\title{
Feedforward Control of Variable Stiffness Joints Robots for Vibrations Suppression
}

\author{
Luigi Biagiotti, Lorenzo Moriello, Claudio Melchiorri
}

\begin{abstract}
This paper presents a new feedforward controller based on a continuous-time finite impulse response filter, designed to minimize the vibrations that usually affect robot manipulators with elastic joints. In particular, Variable Stiffness Joints (VSJ) robots are considered, since they are usually characterized by a very low level of damping which makes the problem of the oscillations quite important. The proposed approach allows to simplify the overall control structure of VSJ robots, which is based on a decentralized control of each servomotor, imposing the desired position and the desired stiffness at each joint, and on a novel feedforward control, filtering the reference signals. After analyzing some of the filter properties and the method for the parameters choice, experimental results on a VSJ robot demonstrate the importance of the proposed filtering action for minimizing vibrations and oscillations.
\end{abstract}

\section{INTRODUCTION}

In the last two decades, the development of service robots close cooperating with humans has driven the designers towards novel mechanical solutions aiming at increasing the mechanical compliance and reducing the apparent inertia of robot manipulators [1]. Unfortunately, an high level of mechanical compliance deteriorates the performance of the plant, in particular with respect to precision. For this reason, in order to solve simultaneously safety and performance issues, Variable Stiffness Actuators (VSAs), which introduce a mechanical compliance in the joint actuation that can be altered via control action, have been proposed [2], [3], [4], [5]. However, the performance of Variable Stiffness Joints (VSJ) robots are still far from those of standard rigid joints manipulators, because of the high order nonlinear dynamics of the system, due to the additional stiffness variation mechanism, and the strongly nonlinear characteristics of VSAs. Moreover, a major problem of VSAs is the very low intrinsic damping that usually characterizes this type of devices, which may cause vibrations and undesired oscillations, [6]. Accordingly, injecting damping into the system is one of the main control goal in this field. Several control approaches for VSJ robots are presented in the literature. While many controllers are conceived for single-joint systems (see [7], [6] among many others), the multi-joint case is treated less frequently. A feedback linearization algorithm is designed and

L. Biagiotti is with the Department of Engineering "Enzo Ferrari", University of Modena and Reggio Emilia, via Pietro Vivarelli 10, 41125 Modena, Italy, e-mail: luigi.biagiotti@unimore.it.

C. Melchiorri and L. Moriello are with the Department of Electrical, Electronic and Information Engineering "Guglielmo Marconi", University of Bologna, Viale del Risorgimento 2, 40136 Bologna, Italy, e-mail: \{claudio.melchiorri\},\{lorenzo.moriello2\}@unibo.it.

This activity has been supported by the University of Bologna, with the "FARB Linea 2" funding action, and by the University of Modena and Reggio Emilia with the "FAR 2015" project. validated in simulations in [8]. A state feedback controller aiming at obtaining the desired level of damping is presented in [9], while, more recently, in [10] a backstepping approach has been proposed to manage the complexity of a VSA system. The same goal, that is dominating the complexity of VSJ robots, is the focus of this work. In this case a multi-dof VSJ robots is built by using variable stiffness servo-motors, QBMove - Maker Pro VSAs by QBRobotics [11]. These actuators are provided with their own control system that allows to achieve a desired position and a desired stiffness of the output shaft with prescribed performance but can not be changed by the user. At most an outer control loop can be built over this basic position/stiffness control. In this paper the use of a feedforward control is preferred for a twofold reason:

- the goal of the control is to cancel the oscillations that affect point-to-point motions of the robot joints, connected to the motors by the (variable stiffness) elastic transmissions with low damping, while static performances, in terms of precision, are not addressed;

- the proposed open-loop control does not alter the stiffness seen at the link side, while a closed-loop control does [6].

In order to achieve these results, a dynamic filter recently proposed in [12], has been considered. Note that, in the literature a number of feedforward controller has been applied to robotic system with elastic elements. In [13], [14], [15] a command shaping technique has been used for robots with flexible links in order to reduce vibrations. The same goal has been achieved for robot manipulators with elastic joints in [16], where an input shaping techniques is combined with an iterative learning mechanism that updates the parameter of a Zero Vibration (ZV) input shaper in order to take into account nonlinear and time-varying characteristics of the plant.

In this paper, after an introduction about the proposed feedforward control for vibration reduction applied to a single joint with elastic transmission, which is a linear time invariant SISO system, a generalization of this method to MIMO systems is given in Sec. III. Then in Sec. IV from the general nonlinear model of a VSJ manipulator the linear approximation of the model for configurations near a nominal operating point is deduced and the parameters of the feedforward control are obtained. Experimental results are reported in Sec. V, while final conclusions are discussed in Sec. VI. 


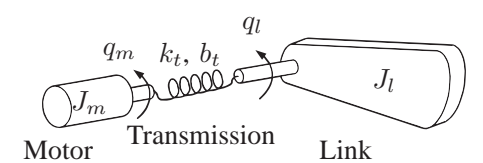

Fig. 1. Structure of a robotic joint with elastic transmission.

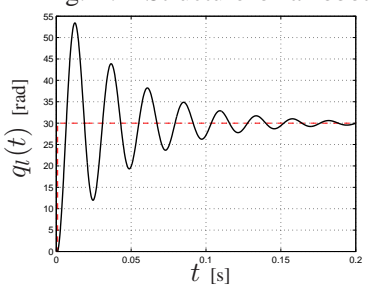

(a)

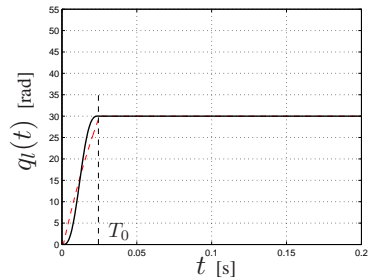

(b)
Fig. 2. Response of the system $G_{m l}(s)$ with $\omega_{n}=260.43 \mathrm{rad} / \mathrm{s}$ and $\delta=0.083$ (the same numerical values used in [12] are considered) to a reference step signal $q_{r e f}(t)$ (a) compared to the response caused by the step filtered by $F_{\exp }(s)$ (b).

\section{FEEDFORWARD CONTROL OF AN ELASTIC JOINT FOR VIBRATIONS SUPPRESSION}

The control of a single robotic joint with elastic transmission, like the one depicted in Fig. 1, can be easily performed by considering only variables at the motor side. It is well known, see e.g. [17], that, in absence of gravity, a PD control based on motor's position $q_{m}$ and velocity $\dot{q}_{m}$ is stable for any positive value of the proportional and derivative gains. Therefore, it is possible to obtain a controlled system that in principle is arbitrarily fast and precise. Unfortunately, even if the motor is able to track the desired reference signal $q_{\text {ref }}(t)$ with small errors (and therefore it is possible to assume $q_{m}(t) \approx q_{\text {ref }}(t)$ ) the link position may be affected by undesired oscillations and vibrations. As a matter of fact, the relationship between the motor position and the link position can be modelled as a second order system, i.e.

$$
G_{m l}(s)=\frac{Q_{l}(s)}{Q_{m}(s)}=\frac{2 \delta \omega_{n} s+\omega_{n}^{2}}{s^{2}+2 \delta \omega_{n} s+\omega_{n}^{2}}
$$

with $\omega_{n}=\sqrt{\frac{k_{t}}{J_{l}}}, \delta=\frac{b_{t}}{2 \sqrt{k_{t} J_{l}}}$, where $Q_{m}(s)=\mathcal{L}\left\{q_{m}(t)\right\}$ and $Q_{l}(s)=\mathcal{L}\left\{q_{l}(t)\right\}$ are the Laplace transforms of the motor and load position. Parameters $J_{l}, b_{t}$ and $k_{t}$ are respectively the link's inertia, the damping and the stiffness of the elastic transmission. The typical response of the system (II) to a step reference input is shown in Fig. 2(a). A simple and effective way to reduce the oscillation consists in applying to the controlled motor a feedforward control action able to properly filter the given reference signal. In [12] it has been shown that the filter

$$
F_{e x p}(s)=\frac{\alpha}{e^{\alpha T_{0}}-1} \frac{1-e^{\alpha T_{0}} e^{-T_{0} s}}{s-\alpha}
$$

guarantees the complete residual vibration ${ }^{1}$ suppression for motion systems with elastic transmission described by (II), fed by step inputs if

$$
\alpha=-\delta \omega_{n}, T_{e}=\frac{2 \pi}{\omega_{n} \sqrt{1-\delta^{2}}} .
$$

${ }^{1}$ The residual vibration is the oscillation that affects the response at the end of motion, which in the case of a step input filtered by $F_{\text {exp }}(s)$ occurs after $T_{0}$ seconds from the application of the step. Note that $F_{\exp }(s)$ has an impulse response of finite duration, equal to $T_{0}$.

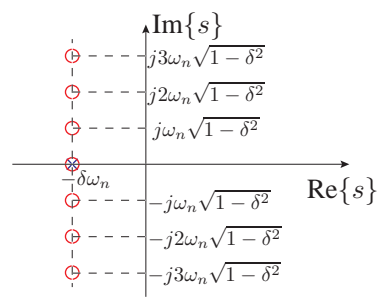

(a)

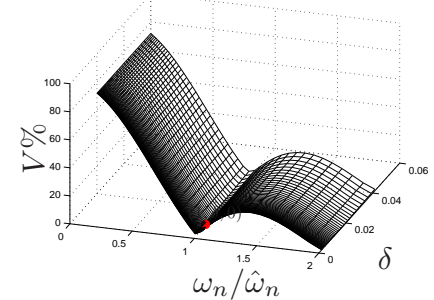

(b)
Fig. 3. Pole-zero map (a) and sensitivity of $F_{\exp }(s)$ to variations in $\omega_{n}$, with respect to its nominal value $\hat{\omega}_{n}$, and $\delta(b)$.

This result, shown in Fig. 2(b), can be proved analytically by considering the step response of the cascade $F_{\text {exp }}(s) G_{m l}(s)$ (see [12] for more details) but it is quite evident by analyzing the poles and zeros of $F_{\text {exp }}(s)$ with the conditions (2). As illustrated in Fig. 3(a), $F_{\text {exp }}(s)$ introduces an infinite number of zeros located along a vertical line in the complex plane. In particular, note that the zeros obtained with $n=1$ exactly cancel the poles of the plant $G_{m l}(s)$. If the parameters of the system $G_{m l}(s)$ are not exactly known, the cancellation will be partial but, in any case, will lead to a reduction of the residual vibration as illustrated in Fig. 3(b), where the percent residual vibration ${ }^{2} V \%$ due to errors in the estimation of the parameters $\omega_{n}$ and $\delta$ is reported. Note that $F_{\text {exp }}(s)$ is more sensitive to variations of the natural frequency $\omega_{n}$ than to the variation of the damping coefficient $\delta$. In any case, by closely analyzing the curve of Fig. 3(b), it results that even an error on the frequency estimation of the $20 \%$ with respect to its nominal value produces a reduction of the vibration of about $80 \%$ with respect to the unfiltered reference input. Therefore, although the parameters of the plant are affected by large uncertainties or are dynamically changing like in the case of a robot manipulator, the filter $F_{\text {exp }}(s)$ leads to a considerable reduction of the vibrations/oscillations.

The considerations reported above allow to generalize the proposed result to any type of Single Input Single Output (SISO) Linear Time-Invariant (LTI) system, characterized by one or more oscillating dynamical modes. Therefore, given a dynamic system modelled as

$$
G(s)=\frac{N(s)}{D(s)\left(s^{2}+2 \delta \omega_{n} s+\omega_{n}^{2}\right)}
$$

where $N(s)$ and $D(s)$ are generic polynomial $(D(s)$ Hurwitz), it is possible to show that the contribution to the response of the oscillating mode characterized by $\left(\delta, \omega_{n}\right)$ can be completely nullified $T_{0}$ seconds after the application of the input signal by inserting between the input and the system a properly tuned filter $F_{\text {exp }}(s)$. Note that the capability of the filter in cancelling residual vibrations does not depend on the particular input considered. Therefore, in order to specify a desired (constant) configuration in lieu of step functions it is possible to assume a smoother signal, such as standard second order trajectories. As shown in Fig. 4, $T_{0}$ seconds after the end of the input trajectory $q_{m}(t)$, supposed

\footnotetext{
${ }^{2}$ According to [18], percent residual vibration is defined as the ratio between the residual vibration to a step command with and without shaping filter.
} 


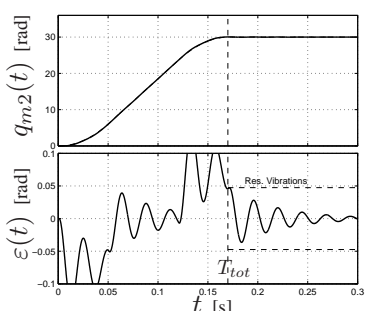

(a)

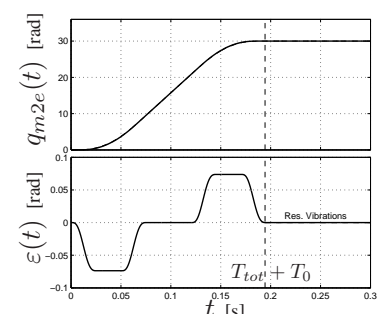

(b)
Fig. 4. Input trajectory $q_{m}(t)$ and related residual vibration $\varepsilon(t)=$ $q_{l}(t)-q_{m}(t)$ of the resonant system of Fig. 2, when applying a second order trajectory $q_{m 2}(t)$ (a) and with the same trajectory filtered by $F_{e x p}(s)$ (b).

of duration $T_{t o t}$, residual vibrations on the system output are completely suppressed. Obviously in this case the amplitude of residual vibration is considerably reduced with respect to the application of step signal because of the use of smooth trajectories.

\section{FEEDFORWARD CONTROL OF MIMO LTI SYSTEMS FOR RESIDUAL VIBRATION SUPPRESSION}

The extension of the results described in Sec. II for SISO systems to Multiple Input Multiple Output (MIMO) systems is straightforward. As a matter of fact, for MIMO LTI systems, usually modelled in the state space domain as

$$
\left\{\begin{array}{l}
\dot{x}=\boldsymbol{A} x+\boldsymbol{B} u \\
y=\boldsymbol{C} x+\boldsymbol{D} u
\end{array}\right.
$$

where $x \in \mathcal{R}^{n}$ is the state vector, $u \in \mathcal{R}^{r}$ is the input vector, $y \in \mathcal{R}^{m}$ is the output vector, and $\{\boldsymbol{A}, \boldsymbol{B}, \boldsymbol{C}, \boldsymbol{D}\}$ are matrices of appropriate dimensions, it is possible to deduce the transfer matrix, i.e. the matrix of the transfer functions between the $r$ inputs and the $m$ outputs,

$$
\boldsymbol{H}(s)=\frac{\boldsymbol{C} \boldsymbol{A d j}\left(s \boldsymbol{I}_{n}-\boldsymbol{A}\right) \boldsymbol{B}+\left|s \boldsymbol{I}_{n}-\boldsymbol{A}\right| \boldsymbol{D}}{\left|s \boldsymbol{I}_{n}-\boldsymbol{A}\right|}
$$

where $\operatorname{Adj}(\boldsymbol{X})$ is the adjoint matrix associated with $\boldsymbol{X}$ and $|\boldsymbol{X}|$ denotes the determinant of $\boldsymbol{X}$. The term $\left|s \boldsymbol{I}_{n}-\boldsymbol{A}\right|$ is an $n$-th polynomial, whose roots are the poles ${ }^{3}$ of the transfer functions that compose $\boldsymbol{H}(s)$. Note that, if no cancellations occur between the numerator and the denominator of these transfer functions, they share the same poles. Therefore, in order to suppress the effects of a poorly damped mode $\left(\delta, \omega_{n}\right)$ on the outputs, it is necessary to insert a filter $F_{\exp }(s)$ before each of the $r$ inputs.

\section{FEEDFORWARd CONTROL OF ROBOTIC MANIPULATORS WITH ELASTIC JOINTS}

In order to apply the technique proposed in sections II and III to a robotic system it is necessary to consider the complete model of the manipulator. The reduced model ${ }^{4}$ of a visco-elastic joints robot is

$$
\begin{aligned}
\boldsymbol{M}\left(q_{l}\right) \ddot{q}_{l}+\boldsymbol{C}\left(q_{l}, \dot{q}_{l}\right) \dot{q}_{l}+\boldsymbol{g}\left(q_{l}\right) & +\boldsymbol{K}_{t} \cdot\left(q_{l}-q_{m}\right) \\
& +\boldsymbol{B}_{t} \cdot\left(\dot{q}_{l}-\dot{q}_{m}\right)=0
\end{aligned}
$$

\footnotetext{
${ }^{3}$ As well-known, if no cancellations occur the poles coincide with the eigenvalues of matrix $\boldsymbol{A}$.

${ }^{4}$ This model is based on the assumption that the angular kinetic energy of the motors is only due to their own spinning [19].
}

where $\boldsymbol{M}\left(q_{l}\right)$, and $\boldsymbol{C}\left(q_{l}, \dot{q}_{l}\right)$ are the inertia and centrifugal/Coriolis forces matrices, $\boldsymbol{g}\left(q_{l}\right)$ is the gravity term, $\boldsymbol{K}_{t}=$ $\operatorname{diag}\left\{k_{t i}\right\}, \boldsymbol{B}_{t}=\operatorname{diag}\left\{b_{t i}\right\}$ are the matrices of the transmission stiffness and viscous friction, $q_{l}$ and $q_{m}$ denote the vector of the joint positions at the link side and at the motor side, respectively [20]. Note that the motors' dynamics that usually accompanies (6) has been neglected since, according to a standard decentralized control of robot manipulators, it is assumed that the motors behave like ideal position sources able to impose any desired configuration $q_{m}$.

The model of VSJ robots can be ideally obtained from (6) by assuming that the stiffness matrix is not a constant but a function of time, i.e. $\boldsymbol{K}_{t}=\boldsymbol{K}_{t}(t)$. The stiffness modification is generally obtained with extra command inputs to the robot system that allow to change each joint stiffness independently, i.e. $k_{t i}=k_{t i}\left(p_{i}\right)$ where $p_{i}$ denotes the activation signal of the stiffness of the $i$-th joint. Therefore, it is possible to rewrite the transmission stiffness matrix as $\boldsymbol{K}_{t}=\boldsymbol{K}_{t}(p)$. In many cases, in particular when the variable stiffness mechanism is obtained with a couple of antagonistic actuators (like in the experiments proposed in this paper) [8], the elastic torque not only depends on the external signal $p(t)$ but it is also a nonlinear function of the motors displacement. As a consequence, in lieu of $\boldsymbol{K}_{t}(p) \cdot\left(q_{l}-q_{m}\right)$ the expression of the elastic transmission torque must be rewritten in a more general way as $\tau_{\text {el }}=\tau_{\text {el }}\left(q_{l}-q_{m}, p\right)$ where $\tau_{\text {el }}(\Delta q, \cdot)$ denotes a vectorial nonlinear function whose elements are odd strictly monotonically increasing functions of $\Delta q$ and $\tau_{\mathrm{el}}(0, \cdot)=0$. Finally, it is worth noticing that often the variable stiffness mechanism makes also the damping torques not constant but variable as a function of the time. Therefore, a quite general expression that describes the dynamics of VSJ robots is

$$
\begin{aligned}
\boldsymbol{M}\left(q_{l}\right) \ddot{q}_{l}+\boldsymbol{C}\left(q_{l}, \dot{q}_{l}\right) \dot{q}_{l}+\boldsymbol{g}\left(q_{l}\right) & +\boldsymbol{\tau}_{e l}\left(q_{l}-q_{m}, p\right) \\
& +\boldsymbol{\tau}_{\text {damp }}\left(\dot{q}_{l}-\dot{q}_{m}, p\right)=0
\end{aligned}
$$

where, similarly to $\tau_{\text {el }}, \tau_{\text {damp }}(\Delta \dot{q}, \cdot)$ denotes a vectorial nonlinear function whose elements are odd strictly monotonically increasing functions of $\Delta \dot{q}$ and $\tau_{\text {damp }}(0, \cdot)=0$.

\section{A. Linearized model of a VSJ robot and feedforward design}

In order to find the parameters of the proposed filter for feedforward control for a given value $p=p^{\star}$, it is necessary to linearize (7) around the desired equilibrium state $\left(q_{l}, \dot{q}_{l}\right)=$ $\left(q_{l}^{\star}, 0\right)$ with $q_{l}^{\star}$ related to the equilibrium input $\left(q_{m}, \dot{q}_{m}\right)=$ $\left(q_{m}^{\star}, 0\right)$ by

$$
\boldsymbol{g}\left(q_{l}^{\star}\right)+\boldsymbol{\tau}_{\mathrm{el}}\left(q_{l}^{\star}-q_{m}^{\star}\right)=0 .
$$

Note that, for the sake of clarity, since the input $p$ is supposed to be a constant the dependance of $\tau_{\text {el }}$ and $\tau_{\text {damp }}$ on it has been omitted. The approximation of (7) by Taylor series expansion up to the first order provides the following expression

$$
\begin{aligned}
& \boldsymbol{M}\left(q_{l}^{\star}\right) \Delta \ddot{q}_{l}+\boldsymbol{g}\left(q_{l}^{\star}\right)+\left.\frac{\partial \boldsymbol{g}\left(q_{l}\right)}{\partial q_{l}}\right|_{q_{l}=q_{l}^{\star}} \Delta q_{l}+\boldsymbol{\tau}_{\mathrm{el}}\left(q_{l}^{\star}-q_{m}^{\star}\right)
\end{aligned}
$$

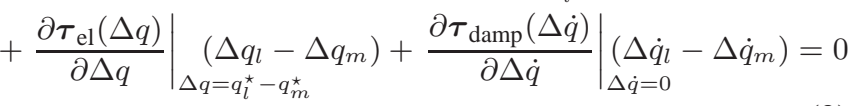


where $\Delta q_{l}=q_{l}-q_{l}^{\star}, \Delta q_{m}=q_{m}-q_{m}^{\star}$, etc. represent small variations with respect to the corresponding equilibrium values. Note that centrifugal/Coriolis terms, that are quadratic with respect to the velocity, disappear in the linearized model. By substituting (8) in (9) and denoting

$$
\begin{gathered}
\boldsymbol{G}^{\star}=\left.\frac{\partial \boldsymbol{g}\left(q_{l}\right)}{\partial q_{l}}\right|_{q_{l}=q_{l}^{\star}}, \boldsymbol{K}_{t}^{\star}=\left.\frac{\partial \boldsymbol{\tau}_{\mathrm{el}}(\Delta q)}{\partial \Delta q}\right|_{\Delta q=q_{l}^{\star}-q_{m}^{\star}}, \\
\boldsymbol{B}_{t}^{\star}=\left.\frac{\partial \boldsymbol{\tau}_{\mathrm{damp}}(\Delta \dot{q})}{\partial \Delta \dot{q}}\right|_{\Delta \dot{q}=0}
\end{gathered}
$$

the expression of the linearized model becomes

$\boldsymbol{M}\left(q_{l}^{\star}\right) \Delta \ddot{q}_{l}+\boldsymbol{G}^{\star} \Delta q_{l}+\boldsymbol{K}_{t}^{\star}\left(\Delta q_{l}-\Delta q_{m}\right)+\boldsymbol{B}_{t}^{\star}\left(\Delta \dot{q}_{l}-\Delta \dot{q}_{m}\right)=0$

which can be rewritten in the state-space form such as (4) with

$$
\begin{aligned}
\boldsymbol{A} & =\left[\begin{array}{cc}
\mathbf{0}_{n} & \boldsymbol{I}_{n} \\
-\boldsymbol{M}^{-1}\left(q_{l}^{\star}\right) \boldsymbol{K}_{t}^{\star}-\boldsymbol{M}^{-1}\left(q_{l}^{\star}\right) \boldsymbol{G}^{\star} & -\boldsymbol{M}^{-1}\left(q_{l}^{\star}\right) \boldsymbol{B}_{t}^{\star}
\end{array}\right] \\
\boldsymbol{B} & =\left[\begin{array}{cc}
\mathbf{0}_{n} & \mathbf{0}_{n} \\
\boldsymbol{M}^{-1}\left(q_{l}^{\star}\right) \boldsymbol{K}_{t}^{\star} & \boldsymbol{M}^{-1}\left(q_{l}^{\star}\right) \boldsymbol{B}_{t}^{\star}
\end{array}\right]
\end{aligned}
$$

where the state and input vectors are $x=\left[\Delta q_{l} \Delta \dot{q}_{l}\right]^{T}$ and $u=\left[\Delta q_{m} \Delta \dot{q}_{m}\right]^{T}$ respectively. By analyzing the eigenvalues of the matrix $\boldsymbol{A}$ it is possible to find the values of the resonant modes that affect the robotic plant. A $n$ degrees-of-freedom robot manipulator with undamped or poorly damped elastic joints will be characterized by $n$ pairs of complex conjugate eigenvalues with $\left(\delta_{i}, \omega_{n i}\right), i=1, \ldots, n$. In order to suppress the oscillations at a constant configuration $q_{l}^{\star}$ it is sufficient to filter the reference signals of the motors, and consequently the motor positions $q_{m}(t)$ supposed to be equal to $q_{r e f}(t)$, with a chain of filters $F_{\exp i}(s)$, one for each mode of the system.

\section{EXPERIMENTAL RESULTS}

The method described in previous sections has been tested on a real soft robotic arm build with QBMove - Maker Pro VSAs by QBRobotics [11]. These actuators implement the concept of variable stiffness servo-motors, i.e. motor units that include also the position/current sensing and the control system allowing the user to command both the position and the stiffness of the output shaft with external signals. For these reason, these actuators are very suitable for rapid prototyping robotic systems with variable stiffness joints [5]. QBMove VSAs are provided with an easy to use Matlab/Simulink toolbox that can run without particular restriction even on standard operating system and communicate with the actuators via USB. In the experiments reported in this section Matlab was running with a fixed step size $T_{s}=2$ ms. For this reason, the filter $F_{\text {exp }}(s)$ has been discretized according to the techniques reported in [12].

The mechanical structure of these VSAs is based on an antagonistic configuration with two servo-motors connected to the output shaft by tendons that are fixed to springs. The working principle is quite simple: the shaft equilibrium position is the mean value of the servos positions and is therefore affected by the concordant motion of the servo-motors, while the stiffness grows as the displacement between the servos increases. Therefore, when the user specifies a give shaft
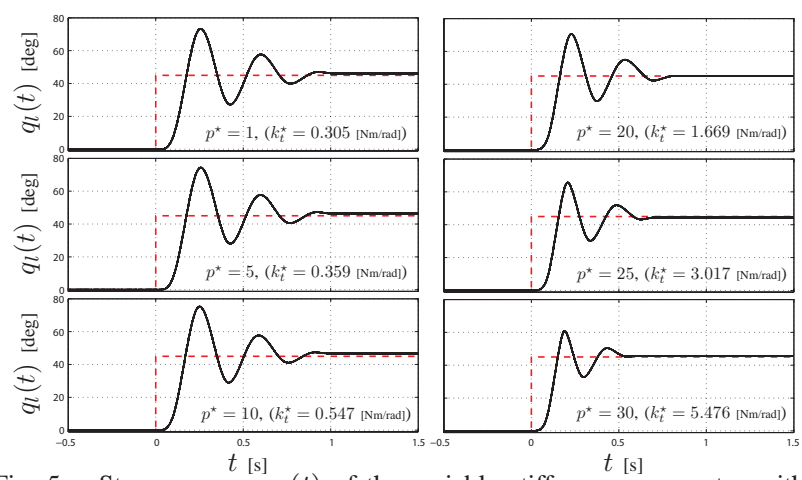

Fig. 5. Step response $q_{l}(t)$ of the variable stiffness servo-motor with an inertial load with different stiffness values $k_{t}^{\star}$. In red the step set-point of $45 \mathrm{deg}$ is reported.

position $q_{l}$ and a stiffness preset $p^{\star}$, these values, related to the motor position by

$$
q_{l}=\frac{q_{m, 1}+q_{m, 2}}{2}, \quad p^{\star}=\frac{q_{m, 1}-q_{m, 2}}{2},
$$

are translated by the QBMove controller in the motor positions $q_{m, 1}$ and $q_{m, 2}$, which are actuated by the two servomotors. As a consequence, a feedforward controller that filters the inputs $q_{l}$ and $p^{\star}$ is actually placed before the motor position $q_{m, 1}$ and $q_{m, 2}$, as supposed in Sec. IV.

\section{A. Characterization of a single actuator}

In order to test the proposed method, an initial experimental analysis on a single actuator has been carried out to estimate the parameters $\alpha$ and $T_{0}$ which characterize the filter $F_{\text {exp }}(s)$. In order to better appreciate the oscillations due to the elastic transmission, a known inertial load represented by an iron disk of diameter $10 \mathrm{~cm}$ and weight $1 \mathrm{~kg}$ has been attached to the actuator shaft. Then a step of 45 deg have been commanded to the actuator with a fixed stiffness preset value and the response has been evaluated.

Several tests has been performed with different stiffness values in order to analyze the related step responses. As it can be seen from the responses of Fig. 5, the system behaves like a second order system. This means that the dominant dynamics is the mechanical dynamics of the inertia with the elastic transmission, while the dynamics of the two servomotors inside the VSA can be neglected. For each stiffness preset $p^{\star}$, and therefore for each values of the stiffness $k_{t}^{\star}$, the values of the damping coefficient $\delta$ and natural frequency $\omega_{n}$ of the system have been determined, and are reported in Fig. 6. It is worth noticing that, as expected, $\omega_{n}$ increases as the stiffness grows but it is also visible a slight increase of $\delta$, due to the frictional effects of the mechanical transmission of the QBMove VSA. Since $J_{l}$ is known, from $\delta$ and $\omega_{n}$ it is possible to immediately deduce the values of the stiffness and damping $\left(k_{t}^{\star}, b_{t}^{\star}\right)$ about the equilibrium point.

In a first stage of this experimental activity, the proposed feedforward control based on the exponential filter $F_{\exp }(s)$ has been applied to a single actuator and its performances have been compared with those of ZVD Input Shapers, which are the most widespread filtering methods for residual vibration suppression, see [21], [22], [23], and which cause the same time-delay of the proposed filter. In order to appreciate 


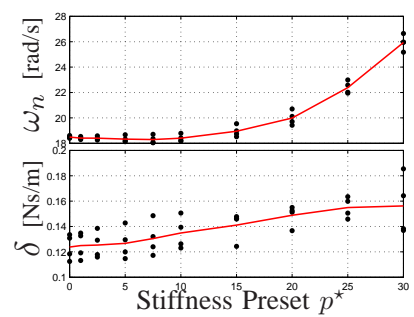

Fig. 6. Estimated parameters $\omega_{n}$ (a) and $\delta$ (b) for different values of stiffness presets $p^{\star}$. Different equilibrium points have been considered.

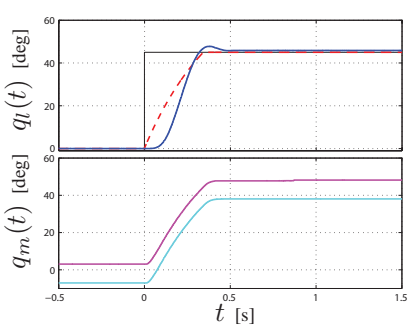

(a)

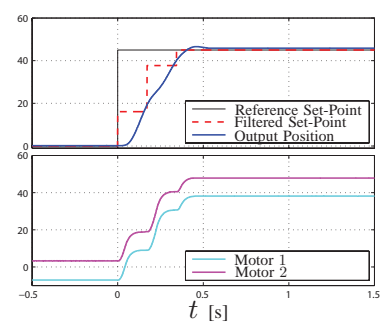

(b)
Fig. 7. Response of the system with stiffness preset $p^{\star}=5$ to a step input of $45 \mathrm{deg}$, shaped by exponential jerk filter (a) and ZVD input shaper (b).

the effectiveness of the proposed method, only very low stiffness values have been considered as they represent a more challenging situation in terms of vibrations. With the parameters derived by means of the procedure described above, the appropriate parameters of the exponential and ZVD filters have been found for every stiffness preset that has been considered. Then the filtered step inputs have been provided to the actuator. The obtained results are shown in Fig. 7: the performances of the two methods in terms of residual vibration reduction and motion time duration are similar and in general very good. However, it is interesting to notice the difference between the motions $q_{m, 1}(t)$ and $q_{m, 2}(t)$ performed by the two servo-motors: while the motors with the ZVD input shaper are fed by several steps, exponential filter provide a smoother trajectory that can be easily tracked.

\section{B. Application of the feedforward control to a planar robot}

The proposed technique has been applied to the 2-dofs planar robotic arm made of QBmove VSAs shown in Fig. 8(a). The actuator parameters $\left(k_{t}^{\star}, b_{t}^{\star}\right)$ derived in previous section for a given stiffness preset $p^{\star}$ have been used to determine the values $\left(\delta_{i}, \omega_{n i}\right)$ of the two vibratory modes that characterize the robot model. Since these values depend on the equilibrium configuration (and in particular on $q_{l, 2}$ ) but their variation is rather limited ${ }^{5}$, the entire range of variation of $q_{l, 2}$ has been considered for a given $p^{\star}$ and the mid value of the interval in which the parameters range has been assumed. In this way, the level of the vibrations is minimized for any possible robot configuration. In Fig. 8(b), this approach is illustrated, with respect to the natural frequencies. From these values the parameters of two exponential filters, which are arranged in a cascade configuration on the reference inputs of the motor, are obtained, see Fig. 9. Also in this case the behavior obtained with the proposed exponential filter

${ }^{5}$ In the example reported in Fig. 8(b), the variations of the natural frequencies are less than $20 \%$.

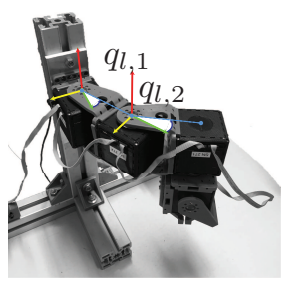

(a)

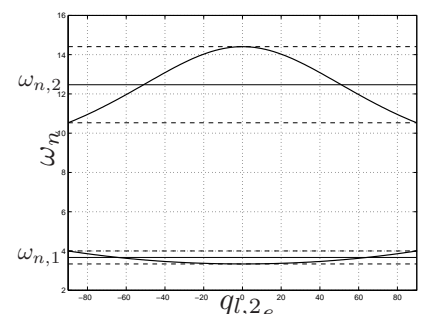

(b)
Fig. 8. Picture of the 2-dofs robotic arm made of QBmove VSAs (a). In (b) theoretical values of $\omega_{n, i}, i=1,2$ for the 2-dofs robot are presented as a function of the equilibrium value $q_{l, 2}$, computed for $p^{\star}=5$.

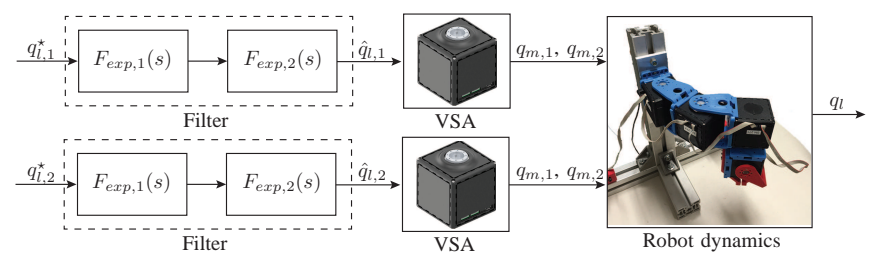

Fig. 9. Block-scheme representation of the feedforward control.

is compared with the one obtained with ZVD Input Shapers. In the test shown in Fig. 10.I, only the first joint is moved, according to a step signal of $30 \mathrm{deg}$. Like in the example of Fig. 8(b), the preset stiffness signal has been set to 5 . Despite the nonlinear behavior of the robot, the cascade of filters, designed for a linear system, is able to cancel the oscillation on the first joint and also to considerably reduce the mutual influence with the second joint, see Fig. 10.I(b). In Fig. 10.II a simultaneous motion of $30 \mathrm{deg}$ of both joints is required. It is quite evident that the proposed method eliminates residual vibrations. Moreover, it guarantees a smoother motion with respect to the ZVD input shaping technique with the same time performance. In both experiments it can be noted a considerable position error due to the fact that feedforward control is not able to compensate for friction effects (the gravity does not affect the system which moves on the horizontal plane but the unbalanced load of the links produces a bending torque on the joint axes that causes a considerable increase of static and Coulomb friction with respect to the case of the disk used as inertia for the single actuator). Anyway, the fact that even without filters the static error is comparable proves that this problem is not related to the specific trajectory generation, but rather to the small value of the stiffness.

In Fig. 10.III the same experiment of Fig. 10.II but with an higher value of the stiffness $\left(p^{\star}=30\right)$ is shown. The conclusions do not change with respect to the previous test, that is the use of exponential filters on the reference inputs cancels the oscillations on the joints positions. In this case, the static precision slightly improves, because of the higher stiffness.

\section{CONCLUSiOnS}

In this paper, a feedforward control based on a chain of exponential filters has been proposed for the suppression of the oscillations that usually affect variable stiffness joints robots. By means of an experimental activity on a simple robotic setup built with commercial VSAs, the proposed method has been proved to be very effective for residual vibration 

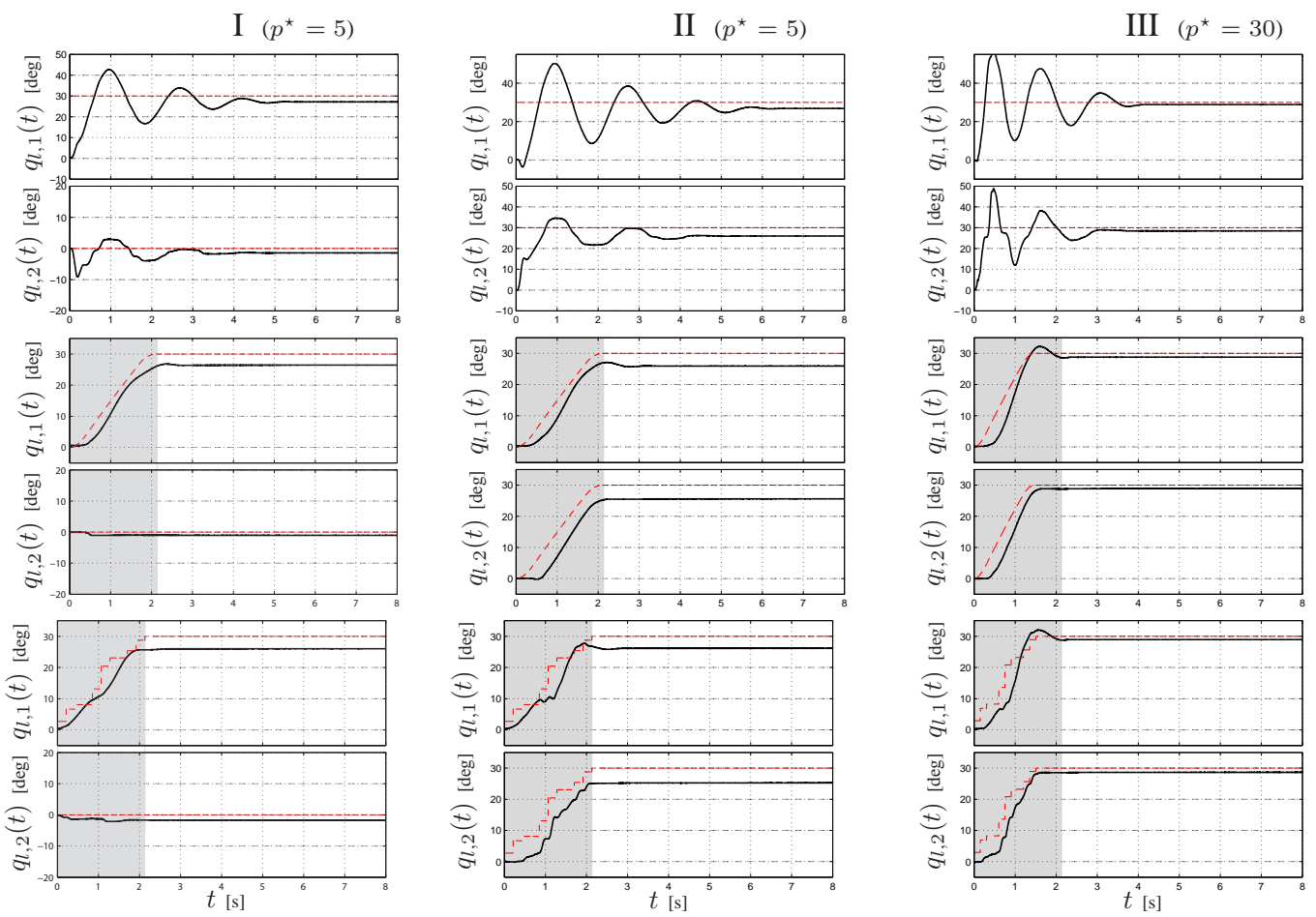

a)

b)

c)

Fig. 10. Response of the actuators $\left(q_{l, 1}(t)\right.$ and $\left.q_{l, 2}(t)\right)$ that compose the 2-dofs robotic arm to a step input trajectory, with a prescribed stiffness preset $p^{\star}$. In dashed red the actual trajectory is reported. In (a) are reported pure step inputs, in (b) the steps are filtered by means of the exponential filter, in (c) the step inputs are shaped using ZVD input shaper.

reduction, even if the problem of static precision still remain. A possible improvement of the proposed technique consists in combining the feedforward control with a mild feedback control, that guarantees small static errors without modifying too much the stiffness seen at the joints side.

\section{REFERENCES}

[1] A. Bicchi, S. Rizzini, and G. Tonietti, "Compliant design for intrinsic safety: general issues and preliminary design," in Intelligent Robots and Systems, 2001 IEEE/RSJ Int. Conf. on, vol. 4, 2001, pp. 18641869 vol.4.

[2] J. Choi, S. Park, W. Lee, and S.-C. Kang, "Design of a robot joint with variable stiffness," in Robotics and Automation (ICRA), 2008 IEEE Int. Conf. on, May 2008, pp. 1760-1765.

[3] S. Wolf and G. Hirzinger, "A new variable stiffness design: Matching requirements of the next robot generation," in Robotics and Automation (ICRA), 2008 IEEE Int. Conf. on, May 2008, pp. 1741-1746.

[4] N. Tsagarakis, I. Sardellitti, and D. Caldwell, "A new variable stiffness actuator (compact-vsa): Design and modelling," in Intelligent Robots and Systems (IROS), 2011 IEEE/RSJ International Conference on, Sept 2011, pp. 378-383.

[5] M. Catalano, G. Grioli, M. Garabini, F. Bonomo, M. Mancinit, N. Tsagarakis, and A. Bicchi, "Vsa-cubebot: A modular variable stiffness platform for multiple degrees of freedom robots," in Robotics and Automation (ICRA), 2011 IEEE Int. Conf. on, May 2011, pp. 5090-5095.

[6] A. Albu-Schäffer, S. Wolf, O. Eiberger, S. Haddadin, F. Petit, and M. Chalon, "Dynamic modelling and control of variable stiffness actuators," in Robotics and Automation (ICRA), 2010 IEEE Int. Conf. on, May 2010, pp. 2155-2162.

[7] G. Tonietti, R. Schiavi, and A. Bicchi, "Design and control of a variable stiffness actuator for safe and fast physical human/robot interaction," in Robotics and Automation (ICRA), 2005 IEEE Int. Conf. on, April 2005, pp. 526-531.

[8] G. Palli, C. Melchiorri, and A. De Luca, "On the feedback linearization of robots with variable joint stiffness," in Robotics and Automation (ICRA), 2008 IEEE Int. Conf. on, May 2008, pp. 1753-1759.

[9] F. Petit and A. Albu-Schäffer, "State feedback damping control for a multi dof variable stiffness robot arm," in Robotics and Automation (ICRA), 2011 IEEE Int. Conf. on, May 2011, pp. 5561-5567.
[10] F. Petit, A. Daasch, and A. Albu-Schaffer, "Backstepping control of variable stiffness robots," Control Systems Technology, IEEE Transactions on, vol. PP, no. 99, pp. 1-1, 2015.

[11] QBMove - maker pro datasheet. [Online]. Available: http://media. wix.com/ugd/45c5a1_792590e00b134129b2b6363a1ea7de45.pdf

[12] L. Biagiotti, C. Melchiorri, and L. Moriello, "Optimal trajectories for vibration reduction based on exponential filters," Control Systems Technology, IEEE Transactions on, vol. PP, no. 99, pp. 1-1, 2015.

[13] A. K. Banerjee and W. E. Singhose, "Command shaping in tracking control of a two-link flexible robot," Journal of Guidance, Control, and Dynamics, vol. 21, no. 6, pp. 1012-1015, 1998.

[14] D. Magee and W. Book, "Optimal filtering to minimize the elastic behavior in serial link manipulators," in American Control Conference, 1998., vol. 5, Philadelphia, PA, USA, 1998, pp. 2637 - 2642.

[15] T. M. Mohamed, Z., "Command shaping techniques for vibration control of a flexible robot manipulator," Mechatronics, vol. 14, no. 1, pp. 69-90, 2004.

[16] J. J. Kim and B. Agrawal, "Experiments on jerk-limited slew maneuvers of a flexible spacecraft," in Guidance, Navigation, and Control and Co-located Conferences. American Institute of Aeronautics and Astronautics, Aug. 2006, pp. - .

[17] A. De Luca and W. Book, Springer Handbook of Robotics. Berlin: Springer Verlag, 2008, ch. Robots with Flexible Elements, pp. 287317.

[18] K. Kozak, W. Singhose, and I. Ebert-Uphoff, "Performance measures for input shaping and command generation," Journal of Dynamic Systems Measurement and Control, vol. 128, no. 3, pp. 731-736, 2006.

[19] M. W. Spong, "Modeling and control of elastic joint robots," Journal of Dynamic Systems Measurement and Control-transactions of The Asme, vol. 104, no. 4, pp. 310-319, 1987.

[20] A. De Luca, R. Farina, and P. Lucibello, "On the control of robots with visco-elastic joints," in Robotics and Automation (ICRA), 2005 IEEE Int. Conf. on, April 2005, pp. 4297-4302.

[21] O. J. M. Smith, "Posicast control of damped oscillatory systems," Proceedings of the IRE, vol. 45, no. 9, pp. 1249-1255, Sept 1957.

[22] N. C. Singer and W. P. Seering, "Preshaping command inputs to reduce system vibration," ASME Journal of Dynamic Systems, Measurement, and Control, vol. 112, pp. 76-82, 1990.

[23] T. Tuttle and W. Seering, "A zero-placement technique for designing shaped inputs to suppress multiple-mode vibration," in American Control Conference, 1994, vol. 3, 1994, pp. 2533 - 2537. 